Radial and Nonradial Pulsations as Probes of Stellar Physics

ASP Conference Series, Vol. 259, 2002

C. Aerts, T.R. Bedding, \& J. Christensen-Dalsgaard, eds.

\title{
Asteroseismology with CORALIE: Results on Procyon and $\beta$ Hydri
}

\author{
F. Carrier, F. Bouchy, F. Kienzle, A. Blecha \\ Observatoire de Genève, CH-1290 Sauverny, Switzerland
}

\begin{abstract}
The spectrograph CORALIE reaches the radial velocity precision needed for an asteroseismological program on bright solar-like stars. We present here two results obtained with this instrument on the stars Procyon and $\beta$ Hydri. A third campaign, on $\alpha$ Cen $\mathrm{A}$, is presented independently in these proceedings by Carrier et al.
\end{abstract}

\section{Spectrograph CORALIE}

The high-resolution fiber-fed echelle spectrograph CORALIE (Queloz et al., 2000 ), mounted on the 1.2-m Swiss telescope at La Silla (ESO, Chile), reaches a resolving power of $50000(\lambda / \Delta \lambda)$. During stellar exposures, a separated reference fiber is continuously illuminated by a Thorium-Argon lamp, in order to monitor the spectrograph stability and thus to obtain high-accuracy velocity measurements. The wavelength coverage of these spectra is $3875-6820 \AA$, recorded on 68 orders. The main limits for an asteroseismological campaign are the small diameter of the telescope and the long CCD read-out time of 100-s.

\section{Asteroseismological campaigns}

Several campaigns have been done with CORALIE on $\alpha$ Cen A (Carrier et al., these proceedings), $\beta$ hydri (Carrier et al., 2001) and Procyon. The results on $\beta$ hydri and Procyon respectively confirm the independent detections of solar-like oscillations announced by Bedding et al. (2001) and by Martic et al. (1999). Two independent instruments and calibration methods obtained very similar power excesses thus leaving little doubt of the stellar origin of the detected signal.

\subsection{Solar-like oscillations in Procyon}

Procyon was observed during 11 nights in February 1999. The resulting 908 high-accuracy radial velocities exhibit an rms scatter of $3.08 \mathrm{~m} \mathrm{~s}^{-1}$. These measurements show significant excess in the power spectrum (Fig. 1) between 0.5-1.5 $\mathrm{mHz}$ with a mean peak amplitude of $0.60 \pm 0.05 \mathrm{~m} \mathrm{~s}^{-1}$. The mean white noise level in the range $2-3 \mathrm{mHz}$ reaches $0.0129 \mathrm{~m}^{2} \mathrm{~s}^{-2}$, namely $0.10 \mathrm{~m} \mathrm{~s}^{-1}$ in amplitude. This noise level corresponds to a velocity accuracy of $1.71 \mathrm{~ms}^{-1}$. The average large splitting $\langle\Delta \nu\rangle$ is $58.7 \pm 1.1 \mu \mathrm{Hz}$. 


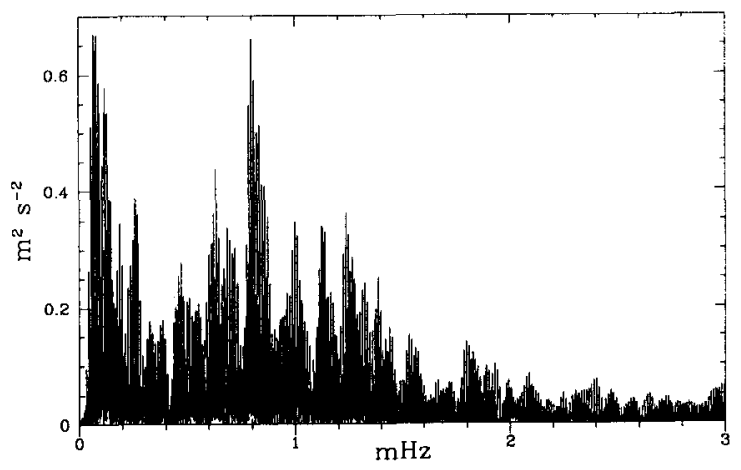

Figure 1. Power spectrum of Doppler shift measurements of Procyon.

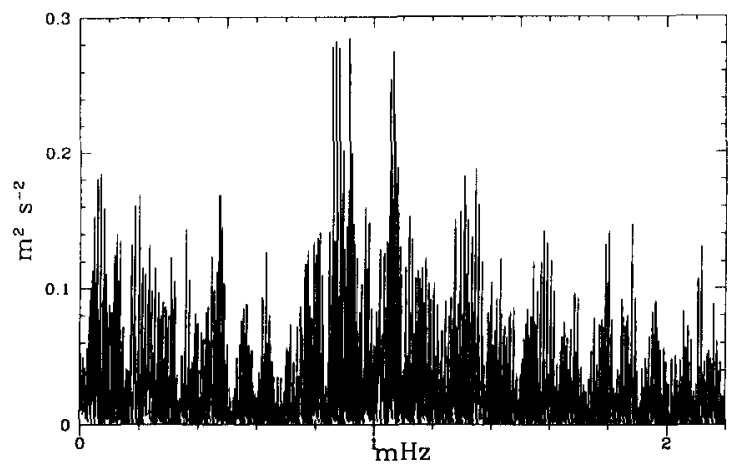

Figure 2. Power spectrum of Doppler shift measurements of $\beta$ Hyi.

\subsection{Solar-like oscillations in $\beta$ Hydri}

$\beta$ Hydri was observed during 2 weeks in June 2000 . The dispersion of the 971 measurements reaches $2.72 \mathrm{~m} \mathrm{~s}^{-1}$. An excess appears in the power spectrum (Fig. 2) between $0.7-1.3 \mathrm{mHz}$ with a mean peak amplitude of $0.40 \pm 0.05 \mathrm{~m} \mathrm{~s}^{-1}$. The mean white noise level in the range $1.6-2.2 \mathrm{mHz}$ reaches $0.019 \mathrm{~m}^{2} \mathrm{~s}^{-2}$, namely $0.12 \mathrm{~m} \mathrm{~s}^{-1}$ in amplitude. This noise level corresponds to a velocity accuracy of $2.15 \mathrm{~m} \mathrm{~s}^{-1}$. The average large splitting $\langle\Delta \nu\rangle$ is $58.0 \pm 1.5 \mu \mathrm{Hz}$.

\section{References}

Bedding, T. R., Butler, R. P., Kjeldsen, H., et al., 2001, ApJ, 549, L105

Carrier, F., Bouchy, F., Kienzle, F., et al., 2001a, A\&A, in press

Martic, M., Schmitt, J., Lebrun, J.-C., et al., 1999, A\&A, 351, 993

Queloz, D., Mayor, M., Weber, L., et al., 2000, A\&A, 354, 99 